\section{Adelantos en la detección de infecciones de transmisión sexual}

Las tasas de incidencia de infecciones de transmisión sexual (ITS) van en aumento en personas de 15 a 25 años. Afortunadamente, ha habido adelantos recientes en el desarrollo de técnicas de tamizaje, detección y prevención de estas enfermedades y sus complicaciones, especialmente de las producidas por Chlamydia trachomatis, los virus del herpes simple y los virus del papiloma humano. Estas técnicas ya se están usando en el contexto de algunas investigaciones, pero su adopción en la práctica clínica suscita un complejo debate. Dos investigadores, en una exhaustiva revisión de artículos publicados de 1998 a 2000, resumen las técnicas que están teniendo mayor influencia en el manejo del paciente con ITS (a excepción de la infección por virus de la inmunodeficiencia humana).

Según sus resultados, Chlamydia trachomatis es el patógeno más detectado como causa de ITS en el mundo desarrollado, y su asociación con el embarazo ectópico y la infertilidad tubaria resalta la importancia del tamizaje de grupos en riesgo de infección. Las pruebas detectoras más utilizadas actualmente son el inmunoensayo enzimático y los ensayos directos a base de sondas de ácidos nucleicos, pero en poblaciones con poca prevalencia de infección el valor predictivo positivo de estas pruebas es bajo, lo cual limita su utilidad para el tamizaje poblacional.

Las pruebas de transcripción y amplificación de ácidos nucleicos, entre ellas las de reacción en cadena de la polimerasa y la ligasa, tienen mayor sensibilidad y especificidad. Poseen la ventaja adicional de que pueden hacerse sin la necesidad de un examen genital, puesto que se pueden efectuar con especímenes de orina siempre que se observe una cadena fría, aunque los resultados son más confiables en hombres que en mujeres. Se han propuesto los siguientes esquemas de tamizaje: a todas las personas que acuden a una clínica, tengan o no síntomas; a todas las mujeres que desean poner fin al embarazo; a jóvenes menores de 25 años sexualmente activos; $y$ a personas mayores de 25 años que han tenido una nueva pareja sexual en el último año. El no tratar a la pareja infectada es quizá la principal causa de fracaso en el tratamiento de la clamidiasis.

Los virus del herpes simple tipo 1 y 2 son los agentes causales del herpes genital. El uso de prue- bas serológicas específicas para diagnosticar la infección por estos virus despierta controversia debido a sus desventajas: los resultados positivos se manifiestan alrededor de 6 semanas después de la exposición; un resultado positivo indica que en algún momento ha habido exposición, pero no necesariamente explica los signos y síntomas por los cuales consulta el paciente; por último, la sensibilidad y especificidad de las pruebas varía de 95 a $99 \%$, de tal modo que en poblaciones donde la prevalencia es baja la mayoría de los resultados positivos serán falsos.

Muchas infecciones herpéticas son asintomáticas, pero se ha observado que la tasa de excreción de virus en personas asintomáticas que son seropositivas y en personas que han tenido síntomas es similar (3 y $2,7 \%$, respectivamente). Como en este último grupo el virus suele eliminarse mientras los síntomas están presentes, se ha especulado que en la fase sintomática la transmisión se podría controlar mediante el uso de condones para proteger la mucosa genital, mientras que el uso continuo de medicamentos antivíricos reduciría el riesgo de transmisión por disminución de la cantidad de virus eliminado durante la fase asintomática y en pacientes sin síntomas. Actualmente estas posibilidades son objeto de estudio, junto con la factibilidad de elaborar una vacuna para la prevención del herpes genital.

Los virus del papiloma humano, los cuales causan más de $90 \%$ de las verrugas genitales, pueden colocarse en distintas categorías según su grado de asociación con el desarrollo de cáncer de cuello uterino. En este sentido, los virus 16 y 18 son los más peligrosos. Actualmente se investiga el uso de diferentes pruebas de detección a base de ADN, y la sensibilidad de estas pruebas, incluido el Hybrid Capture II Assay (Digene, Gaithersburg, Maryland, EE.UU.), es de alrededor de $90 \%$. Su especificidad, sin embargo, plantea un problema, ya que la frecuencia de resultados positivos falsos es cercana a $20 \%$. Hace falta efectuar más estudios para determinar si el tamizaje es factible y económicamente ventajoso.

Los tratamientos de la verrucosis genital siguen siendo poco eficaces y se acompañan de una elevada tasa de recurrencia. El imiquimod, nuevo fármaco inmunógeno, tiene igual eficacia que los otros pero tiene una tasa de recurrencia menor. Se está investigando el desarrollo de una vacuna contra los virus del papiloma humano, tanto por su 
efecto en la prevención de las verrugas genitales como del cáncer cervicouterino, y una vacuna por fusión contra el virus del papiloma humano tipo 6 denominada L2E7 ha mostrado ser inocua e inmunógena, pero en ensayos efectuados hasta ahora no ha sido eficaz para la prevención de las verrugas genitales. Para pacientes con cáncer cervicouterino, las investigaciones actuales se centran en las oncoproteínas E6 y E7. (Gilson RJ C, Mindel A. Recent advances: sexually transmitted infections. BMJ 2001; 322:1160-1164.)

\section{Los inhibidores de la enzima convertidora de la angiotensina reducen el riesgo de enfermedades cardiovasculares en diabéticos}

Las enfermedades cardiovasculares representan la principal causa de mortalidad en diabéticos, debido, entre otros factores, a la alta prevalencia de hipertensión arterial en estos pacientes. Datos recientes también revelan que las personas hipertensas son más propensas a la diabetes mellitus que las personas sin hipertensión. Indican, además, que hasta $75 \%$ de la frecuencia de enfermedades cardiovasculares en diabéticos se puede atribuir a la hipertensión. Se impone, por lo tanto, la necesidad de combatir la hipertensión en estas personas, procurando lograr una presión arterial inferior a 130/85 $\mathrm{mm} \mathrm{Hg}$. Otros factores de riesgo importantes de enfermedad cardiovascular son la obesidad, la aterosclerosis, las dislipemias, la microalbuminuria, la disfunción endotelial, la hiperagregabilidad plaquetaria, las anormalidades de la coagulación y la cardiopatía diabética, entidad que afecta al miocardio, independientemente de los trastornos macro y microvasculares propios de la diabetes, y que explica una buena fracción de la mortalidad por enfermedades cardiovasculares en personas diabéticas.

Un grupo de investigadores en Nueva York hizo una revisión de las últimas investigaciones sobre la diabetes, la hipertensión y las enfermedades cardiovasculares y elaboró un resumen actualizado de los hallazgos. Los estudios apuntan hacia un posible efecto directo de los inhibidores de la enzima convertidora de la angiotensina (ECA) en el corazón y los vasos sanguíneos, además de su efecto sobre la presión arterial. Al parecer, el tratamiento con los inhibidores de la ECA podría mejorar la sensibilidad de estos pacientes a la insulina y retrasar el desarrollo de diabetes en personas en alto riesgo de padecer la enfermedad.

Se ha confirmado que el control riguroso de la presión arterial, a niveles menores de 130 y $85 \mathrm{~mm}$ $\mathrm{Hg}$ para la presión sistólica y diastólica, respectivamente, reduce notablemente la frecuencia de infarto, accidente cerebrovascular y nefropatía terminal en pacientes diabéticos. Se ha demostrado que los inhibidores de la ECA retrasan la progresión de la nefropatía y de las enfermedades cardiovasculares en pacientes diabéticos normotensos. Incluso es posible que ayuden a prevenir la diabetes por interferencia con el mecanismo de la renina-angiotensina. Este descubrimiento, basado en el Heart Outcomes Prevention Evaluation (HOPE), tiene importancia capital, y la administración de los inhibidores de la ECA debe ser parte integral de cualquier tratamiento antihipertensivo en pacientes diabéticos. (Sowers JR, Epstein M, Frohlich ED. Diabetes, hypertension, and cardiovascular disease: an update. Hypertension. 2001;37(4):1053-1059.)

\section{Posibles estrategias de vacunación contra la esquistosomiasis}

La esquistosomiasis, segunda enfermedad parasítica más frecuente en el mundo después de la malaria, afecta a 200 millones de personas. Se trata de una enfermedad crónica debilitante cuyos síntomas obedecen a la acumulación de millones de huevos en los distintos tejidos del cuerpo. La vacunación es uno de los componentes esenciales del control de esta enfermedad.

Investigadores del Instituto Pasteur en Lille, Francia, han dedicado 20 años a identificar los mecanismos inmunológicos que se activan en pacientes con esquistosomiasis, y siete de sus estudios confirman por primera vez que las citoquinas Th2, junto con los anticuerpos IgE, ofrecen protección contra esta parasitosis. La identificación y clonación molecular de un antígeno blanco, una transferasa de la glutationa S (GST), ha permitido demostrar la posibilidad de elaborar una vacuna aprovechando estos conocimientos. En roedores, ganado y primates se ha logrado confirmar la eficacia de dicha vacuna, particularmente su capacidad para reducir la fecundidad de las hembras y la viabilidad de los óvulos mediante la producción de anticuerpos neutralizantes (IgA e IgG).

Ya están en marcha algunos ensayos de fases I y II usando una vacuna a base de Schistosoma haematobium. Esta ha provocado la producción de títulos muy altos de anticuerpos neutralizantes y de citoquinas Th2, tal como se había previsto sobre la base de modelos experimentales. Estos resultados parecen apuntar a que en un plazo relativamente corto será factible elaborar una vacuna eficaz contra la esquistosomiasis. (Capron A, Capron M, Dombrowicz D, Riveau $\mathrm{G}$. Vaccine strategies against schistosomiasis: from concepts to clinical trials. Int Arch Allergy Immunol 2001;124(1-3):9-15.) 


\section{¿Sirven los remedios naturales para aliviar los síntomas de la menopausia?}

El Colegio Estadounidense de Obstetras y Ginecólogos (ACOG) acaba de publicar un boletín sobre el uso de los remedios herbolarios para tratar los síntomas de la menopausia. Esta iniciativa obedece al hecho de que se observa un aumento inusitado del número de mujeres que acuden a estos remedios como sustitutos del tratamiento de restitución hormonal (TRH). Según la ACOG, puede ser que algunos tengan eficacia contra ciertos síntomas, pero estas sustancias no están sujetas a ninguna regulación obligatoria, con el resultado de que carecen de uniformidad. El consumidor no puede estar seguro de la verdadera eficacia y seguridad de ningún producto en particular, ni operan mecanismos que lo protejan contra su contaminación, adulteración o identificación errónea. De hecho, muchos de los productos que se anuncian como "sustitutos" del TRH no ofrecen ningún beneficio sanitario comprobado.

Es alarmante el hecho de que menos de la tercera parte de las mujeres menopáusicas eligen la TRH por no creer en su eficacia o por temor a sus efectos secundarios o al riesgo de desarrollar cáncer de mama. Estas mujeres suelen creer que los remedios naturales son más sanos y eficaces, aunque hasta ahora no haya estudios que lo confirmen. Entre otras cosas, pueden producirse reacciones peligrosas entre estos productos naturales y algunos medicamentos.

El nuevo documento de la ACOG recomienda a los ginecólogos que proporcionen a sus pacientes la siguiente información, basada en una revisión de los pocos estudios que se han efectuado hasta el momento.

- La soja y las isoflavonas pueden aliviar transitoriamente (2 años o menos) los síntomas de la menopausia, tales como los bochornos y la sudación nocturna. Deben consumirse en cantidades moderadas; en exceso, pueden interactuar con los estrógenos y ser nocivas para las mujeres con antecedentes de cánceres dependientes de estrógenos y quizá para otras también.

- La cimicífuga o hierba sonajero puede aliviar los bochornos y la sudación nocturna en el corto plazo (6 meses o menos).

- Debido a su falta de biodisponibilidad, las hormonas que se encuentran en las batatas silvestre y mexicana no deben tener ningún efecto terapéutico. Los extractos de la batata silvestre no son análogos del estrógeno ni de la progesterona. No hay estudios publicados que demuestren la eficacia de la pomada a base de batata silvestre.
- Los beneficios del Dong Quai, hierba que proviene de la China, no son sustentados por las pruebas obtenidas hasta el momento. Puede ser tóxica y hay indicios de que puede aumentar el riesgo de cánceres cutáneos por exposición a los rayos solares.

La ACOG recomienda a los ginecólogos que le pregunten a cada paciente si consume productos naturales y suplementos dietéticos y que los anote en la ficha clínica. Cualquier resultado adverso también debe anotarse y notificarse a la Administración de Alimentos y Medicamentos si el caso se produce en Estados Unidos. A la paciente se le debe indicar que suspenda el tratamiento.

\section{La eliminación de la lepra aún elude a algunos países}

Hace alrededor de un decenio, se fijó la meta de eliminar la lepra del mundo entero. Esa meta hoy en día ha sido alcanzada, según un informe que acaba de emitir la Organización Mundial de la Salud (OMS). Esto significa que desde 1991, la prevalencia de la lepra se ha reducido en más de $90 \%$.

Este hito representa la eliminación de una de las enfermedades más temidas de la humanidad, y una de las que más se acompañan de desfiguración física, estigmatización y rechazo. Se debe, en gran medida, al aumento del acceso a pruebas diagnósticas en etapa temprana de la enfermedad y al tratamiento gratuito en comunidades en mayor riesgo, a su vez parte de una iniciativa lanzada en 1999 y conocida por Alianza Mundial para la Eliminación de la Lepra. A la cabeza de esta iniciativa se encuentran los programas nacionales de los principales países con enfermedad endémica, la OMS, la Fundación Nippon, la Federación Internacional de Asociaciones contra la Lepra (ILEP), los laboratorios Novartis y la Fundación Novartis para el Desarrollo Sostenible, el Fondo Danés de Asistencia para el Desarrollo Internacional (DANIDA) y el Banco Mundial.

Pese a las implicaciones de este logro extraordinario, sigue habiendo seis países con una carga relativamente grande de lepra donde aún es preciso continuar la lucha contra la enfermedad: Brasil, India, Madagascar, Mozambique, Myanmar y Nepal. Estos países enfrentan el reto de proporcionar acceso gratuito al tratamiento medicamentoso múltiple a comunidades que hasta ahora no han sido beneficiadas debido a la baja cobertura de los servicios para el tratamiento de personas leprosas, la falta de infraestructura y el aislamiento impuesto por problemas bélicos. A este efecto se están llevando a cabo grandes campañas de información en 
zonas de alta prevalencia, de tal manera que los pacientes de lepra y su familia se sientan alentados a acudir a un servicio de salud a recibir el tratamiento.

El tratamiento medicamentoso múltiple es, afortunadamente, muy eficaz. Detiene la transmisión de la enfermedad desde la primera dosis y previene la discapacidad. En los últimos 15 años, cerca de 11 millones de leprosos han sido curados con este tratamiento. (Organización Mundial de la Salud. Leprosy: global target attained: remaining endemic countries pose greatest challenge. Comunicado de prensa WHA/2, 16 de mayo de 2001.)

\section{¿Qué frecuencia deben tener las consultas prenatales?}

Según un informe reciente de la Organización Mundial de la Salud, en países en desarrollo las mujeres sanas cuyos embarazos son de bajo riesgo no necesitan consultar al médico ocho veces o más durante la gestación, como suele recomendarse. Los resultados de un estudio no revelaron diferencia alguna entre la frecuencia de hijos con bajo peso al nacer, anemia durante el embarazo, o estancia hospitalaria después del parto en mujeres que acudieron a menos de ocho consultas y a ocho consultas o más. Un menor número de consultas prenatales podría representar un ahorro en países de escasos recursos.

El estudio se basó en una muestra de casi 24.500 mujeres en Arabia Saudita, Argentina, Cuba y Tailandia. Estas mujeres fueron divididas en dos grupos: las primeras acudieron al médico de tres a seis veces durante el embarazo y las segundas, de ocho a once veces. A las mujeres en quienes se determinó la presencia de un embarazo riesgoso, que constituyeron $20 \%$ de la muestra, se les remitió a un especialista o se les trató en consecuencia con su estado delicado. Al terminar el estudio, los investigadores hallaron que las mujeres en ambos grupos tuvieron tasas similares de hospitalización, igual estancia hospitalaria y tasas similares de hijos de bajo peso al nacer, anemia durante el embarazo e infecciones urinarias.

Se observó una frecuencia ligeramente mayor de preeclampsia en las mujeres que tuvieron menos consultas prenatales que en las que acudieron al número habitual de consultas $(1,7 \%$ frente a $1,4 \%$, respectivamente). Cerca de $73 \%$ de las primeras expresaron sentirse satisfechas con el número de consultas prenatales, frente a $84 \%$ de las segundas. No obstante, en países en desarrollo se podrían implantar nuevas pautas sin mayor resistencia por parte de las mujeres o de los proveedores de servicios de salud, aunque sería prudente empezar con una reducción modesta del número de consultas. Lo importante es conducir cada consulta adecuadamente, prestando atención a los aspectos importantes de la gestación. Si llegara a producirse una complicación, se podrían llevar a cabo consultas adicionales. (Mulvihill K. Fewer doctor visits OK for expectant moms: study. Lancet 2001;357(1546): 1551-1564.)

\section{Una dieta sana y el ejercicio pueden reducir el riesgo de diabetes tipo 2}

Mientras más cambian las personas su estilo de vida, más se protegen contra el riesgo de diversas enfermedades, entre ellas la diabetes mellitus. En otras palabras, mejor es hacer pocos cambios que ninguno. La pérdida de peso, por ejemplo, no parece ser componente esencial de la ecuación que favorece la salud. Si bien es cierto que una pérdida de peso moderada reduce mucho el riesgo de diabetes, según los resultados de un estudio finlandés, hacer ejercicio cuatro horas a la semana, aun sin mayor pérdida de peso, lleva a una reducción del riesgo de diabetes. Esto se aplica a cualquier tipo de ejercicio físico, bien sea un deporte, trabajo doméstico, jardinería o ejercicio ocupacional.

El estudio se realizó en 522 adultos de edad mediana con exceso de peso y con una tolerancia alterada a la glucosa, signo precursor de la diabetes. Los pacientes se dividieron en dos grupos. Uno de ellos fue sometido a un programa de dieta y ejercicio, con orientación individual sobre cómo comer sanamente (con menos grasa y más alimentos fibrosos), perder peso y hacer ejercicio. Este grupo se reunió con un nutricionista siete veces durante el primer año y cada tres meses posteriormente. El segundo grupo, que fue el grupo testigo, solamente recibió información escrita sobre los beneficios de una dieta sana y de hacer ejercicio, pero no participó en ningún programa específico.

Al cabo de dos años, las personas en el primer grupo perdieron casi 8 libras, en promedio, mientras que las del segundo perdieron apenas un promedio de 2 libras. A los tres años, 27 personas (3\% al año) en el primer grupo y 59 (6\% al año) en el segundo habían desarrollado diabetes, lo cual representa una reducción del riesgo de $58 \%$ en las personas sometidas a la intervención.

Cambiar los hábitos alimentarios y hacer más ejercicio son tareas difíciles, especialmente para personas obesas y sedentarias, pero cualquier cambio del estilo de vida, por pequeño que sea, aporta un beneficio. El estudio no tuvo suficiente duración para determinar cuánto tiempo los participantes fueron capaces de adherirse a su nuevo estilo de vida, lo 
cual es importante en vista de que la mayoría de las personas vuelven a engordar después de participar en un programa para bajar de peso. No obstante, si los resultados de este estudio son repaldados por los de futuras investigaciones, habrá motivo para contemplar la posibilidad de ofrecer programas del tipo aquí descrito en el contexto de la atención primaria y bajo la cobertura del seguro de salud. (Tataranni PA, Bogardus C. Changing habits to delay diabetes. $N$ Engl J Med 2001;344(18):1343-1350.)

\section{Relación coste-eficacia del tratamiento antirretrovírico combinado en la infección por VIH}

Durante la última década se han producido importantes cambios en el tratamiento de la enfermedad por virus de la inmunodeficiencia humana (VIH). El tratamiento antirretrovírico combinado (TARC) ha reducido la morbilidad y la morbilidad de la infección avanzada. En la actualidad, en los Estados Unidos de América se recomienda el tratamiento con al menos tres antirretrovíricos. Aunque estos tratamientos son muy caros, los estudios observacionales iniciales indicaron que el TARC puede tener una aceptable relación entre los costes y los beneficios, o incluso proporcionar un ahorro. Sin embargo, en estos estudios no se incluyeron los resultados de los principales ensayos clínicos, no se tuvo en cuenta el efecto de la carga vírica sobre la progresión de la enfermedad y no se investigaron las consecuencias del inicio del tratamiento en diferentes estadios de la infección. Los autores de este estudio crearon un modelo global de enfermedad por VIH que toma en consideración los recuentos de células $\mathrm{CD} 4$ y la carga vírica como factores predictivos de la progresión de la enfermedad. Utilizando los datos de varios ensayos clínicos, determinaron el efecto clínico, el coste y la relación coste-eficacia de varias estrategias para el tratamiento de la enfermedad por VIH.

Para comparar diferentes estrategias terapéuticas, se utilizó un modelo informático de enfermedad por VIH en el que se incluyeron las probabilidades mensuales de acontecimientos clínicos tales como modificaciones del recuento de células CD4 y de la carga vírica, aparición de enfermedades oportunistas, reacciones adversas a las medicaciones y muerte. Las medidas de eficacia analizadas fueron las infecciones oportunistas primarias y recurrentes, la esperanza de vida, la esperanza de vida ajustada en función de la calidad de vida y el coste del tratamiento. Los beneficios de los regímenes terapéuticos alternativos se compararon calculando la variación de la razón coste-eficacia, definida como el coste adicional de un régimen en particular, dividido por su beneficio clínico adicional en años de vida (o años de vida ajustados en función de la calidad de vida: AVAC) ganados.

Las infecciones oportunistas consideradas fueron la neumonía por Pneumocystis carinii, la toxoplasmosis, la infección por microorganismos del complejo Mycobacterium avium, las infecciones fúngicas diseminadas y la infección por citomegalovirus. Las causas de muerte se clasificaron como debidas a infecciones oportunistas, SIDA crónico o causas no relacionadas con la infección por VIH. La progresión de la enfermedad por $\mathrm{VIH}$, los riesgos de acontecimientos clínicos, los efectos de los tratamientos y el uso de recursos sanitarios se relacionaron con el recuento de células CD4 (seis categorías: >500, 301-500, 201-300, 101-200, 51-100 y $0-50$ células $/ \mathrm{mm}^{3}$ ) y la carga vírica (cinco categorías $>30000,10$ 001-30 000, 3 001-10 000, 501-3 000 $\mathrm{y} \leq 500$ copias $/ \mathrm{mL}$ ).

Utilizando la simulación de Monte Carlo, se modelaron individualmente las evoluciones clínicas de 1 millón de pacientes hipotéticos. Para el análisis primario, las características de cada paciente (edad, sexo, recuento de células CD4 y carga vírica) se asignaron de forma aleatoria a partir de los datos de un ensayo clínico (el estudio 320 del AIDS Clinical Trials Group: ACTG). Como los pacientes de este estudio tenían enfermedad avanzada, en los análisis secundarios también se utilizaron los datos de otros tres ensayos clínicos (el Johns Hopkins HIV Clinic cohort study; el Italy, the Netherlands, Canada, and Australia (INCAS) trial y el Dupont 006 trial).

Basándose en los resultados del estudio 320 del ACTG, la esperanza de vida de un paciente no tratado fue de 1,97 años (1,53 AVAC), y el coste total de US\$ 45 460. Con la triterapia, las cifras correspondientes fueron de 3,51 años (2,91 AVAC), el coste total de US\$ 77300 , y el coste adicional por AVAC, de US $\$ 23$ 000. Basándose en los resultados de los otros tres ensayos clínicos, las esperanzas de vida fueron mayores, hecho que refleja los mayores recuentos iniciales de células CD4. Aunque los costes médicos totales aumentaron en todos los estudios a medida que lo hacía la supervivencia, las razones coste-eficacia se mantuvieron estables, oscilando entre US\$ 13000 y 23000 por AVAC ganado con el TARC, en comparación con la ausencia de tratamiento.

Utilizando los datos del estudio 320 del ACTG, tanto la esperanza de vida ajustada en función de la calidad de vida como los costes totales aumentaron a medida que lo hacía el recuento inicial de células CD4. Sin embargo, las razones costeeficacia adicionales de la triterapia, comparadas con 
las de la ausencia de tratamiento, se mantuvieron en un margen relativamente estrecho de US\$ 14000 a 26000 por AVAC ganado. El análisis de la eficacia y costes de la biterapia con base en los datos del estudio 320 del ACTG, del ensayo clínico INCAS y del ensayo clínico Dupont 006 reveló que este tratamiento era superior a la ausencia de tratamiento, pero menos eficaz y con peor relación coste-eficacia que la triterapia.

En comparación con la ausencia de tratamiento, el inicio de la triterapia cuando el recuento de células CD4 era de $500 / \mathrm{mm}^{3}$, en presencia de una carga vírica $>30000$ copias $/ \mathrm{mL}$, incrementó los costes totales de US\$ 64210 a 90980 e incrementó la esperanza de vida ajustada en función de la calidad de vida de 5,10 a 6,94 años, con un coste adicional de US\$ 15000 por AVAC ganado. Esta estrategia fue más eficaz y proporcionó un uso más eficiente de los recursos que el inicio del tratamiento cuando el recuento de células CD4 era de $350 / \mathrm{mm}^{3}$. Si se esperaba a que los recuentos de células CD4 fueran de $200 / \mathrm{mm}^{3}$ para iniciar el tratamiento, los costes totales aumentaban y la esperanza de vida ajustada en función de la calidad de vida disminuía.

Así pues, los resultados obtenidos revelaron que, en comparación con la ausencia de tratamiento, la triterapia incrementa la esperanza de vida ajustada en función de la calidad de vida en 1,38 a 2,67 años, con razones coste-eficacia que oscilan entre US\$ 13000 y 23000 por AVAC ganado. Estos aumentos de la esperanza de vida constituyen una mejoría clínicamente significativa y son similares a los beneficios proporcionados por el tratamiento trombolítico en pacientes con sospecha de infarto agudo de miocardio (aumento de la esperanza de vida de 1,25 años). La razón coste-eficacia del TARC no es tan buena como la de la profilaxis frente a la neumonía por P. carinii (US\$ 2300 por AVAC ganado), pero sí mejor que la de la profilaxis frente a la infección por microorganismos del complejo M. avium (US\$ 31000 por AVAC ganado). A pesar de ser caro, el tratamiento antirretrovírico tiene mejor relación coste-eficacia que muchos tratamientos frente a otras enfermedades, como la radioterapia del cáncer de mama en estadio temprano (US\$ 30000 por AVAC ganado), el tratamiento de la hipercolesterolemia (US\$ 47000 por AVAC ganado) o la diálisis en pacientes con menos de 6 meses de esperanza de vida (US\$ 150000 por AVAC ganado).

Aunque este análisis tiene sus limitaciones, los resultados obtenidos indican que la triterapia antirretrovírica tiene una buena relación coste-eficacia y debería ser ofrecida a todos los pacientes que pudieran beneficiarse de ella. Asimismo, indican que parece razonable iniciar el tratamiento con recuentos de células CD4 más elevados que los tradicionalmente aceptados. (Freedberg KA, Losina E, Weinstein MC, Paltiel AD, Cohen CJ, Seage GR et al. The Cost Effectiveness of Combination Antiretroviral Therapy for HIV Disease. N Engl J Med 2001;344:824-831)

\section{Se detectan diferentes mecanismos de resistencia a la cloroquina en dos especies de Plasmodium}

La resistencia a la cloroquina en Plasmodium vivax se notificó por vez primera en 1989, treinta años después de que se descubriera resistencia a la cloroquina en $P$. falciparum. Actualmente, la prevención y el tratamiento de la malaria causada por ambas especies se ven comprometidos por la creciente frecuencia de resistencia a este fármaco, durante largas décadas usado como elemento clave en el control de la malaria.

Hace poco se demostró que algunas mutaciones de una proteína de la vacuola digestiva codificada por el gen $p f c r t$, de 13 exones, desempeñan un papel central en la resistencia de $P$. falciparum a la cloroquina. Lo que aún no se había determinado es si esta misma mutación era la reponsable de la resistencia en otras especies de Plasmodium, como $P$. vivax. Con este fin, un grupo de investigadores de los Institutos Nacionales de Salud de los Estados Unidos de América estudió los homólogos del gen pfcrt de P. vivax, P. knowlesi, P. berghei y Dictyostelium discoideum y observó una correspondencia genómica entre $P$. falciparum y $P$. vivax. Sin embargo, al examinar parásitos aislados de pacientes y líneas parasitarias adaptadas de monos, no detectaron ninguna asociación entre la resistencia a la cloroquina in vivo y las mutaciones de codones del gen de $P$. vivax. Los autores sacan en conclusión que distintos fenómenos moleculares explican la resistencia de $P$. vivax y $P$. falciparum a la cloroquina. (Nomura T, Carlton Jane M-R, Baird JK, del Portillo HA, Fryauff DJ, Rathore D, et al. Evidence for different mechanisms of chloroquine resistance in 2 Plasmodium species that cause human malaria. J Infect Dis 2001;183:1653-1661.)

\section{Revisión sistemática sobre el empleo de mucolíticos orales en las exacerbaciones de la neumopatía obstructiva crónica}

Al menos la mitad de los fumadores acaban sufriendo bronquitis crónica y cerca del $15 \%$ padecen síntomas incapacitantes relacionados con la neumopatía obstructiva crónica. Los pacientes con 
bronquitis crónica o neumopatía obstructiva crónica pueden sufrir repetidas exacerbaciones que incrementan la morbilidad y los costes de la atención de salud. Aunque estos episodios pueden ser tratados con antibióticos o corticoesteroides, sería conveniente disponer de otros tratamientos que redujeran la frecuencia y duración de las exacerbaciones agudas. En algunos países europeos los mucolíticos se encuentran entre los fármacos más prescritos con este fin en pacientes con bronquitis crónica, pero en el Reino Unido y Australia su empleo es mucho menos frecuente porque generalmente se consideran ineficaces, y carecen de financiación pública cuando son recetados a adultos con neumopatía osbtructiva crónica.

Los autores del presente estudio realizaron una revisión sistemática de los ensayos clínicos publicados, con el fin de determinar si el tratamiento con mucolíticos en pacientes con bronquitis crónica o neumopatía obstructiva crónica reduce la frecuencia de las exacerbaciones y los días de enfermedad, si mejora la función pulmonar o si aumenta los acontecimientos adversos.

Para identificar los estudios sobre el tema se efectuó una búsqueda en el registro del Cochrane Airways Group. En la búsqueda se utilizaron las siguientes palabras clave: (chronic bronchitis OR chronic obstructive pulmonary disease) AND (mucolytics OR N-acetylcysteine OR bromhexine OR S-carboxymethylcysteine OR ambroxol OR sobrerol OR iodinated glycerol). Para ser incluidos en la revisión, los estudios tenían que ser estudios aleatorizados, doblemente enmascarados y controlados con placebo sobre el empleo de mucolíticos orales al menos durante 2 meses. Se excluyeron los estudios en los que se utilizaron mucolíticos inhalados o combinaciones de mucolíticos y antibióticos, broncodilatadores, desoxirribonucleasas o proteasas. Los participantes tenían que ser adultos (> 20 años) con bronquitis crónica o neumopatía obstructiva crónica, definidas según criterios ampliamente aceptados. Se excluyeron los estudios realizados en pacientes con asma o fibrosis quística. Las medidas de eficacia primarias fueron el número de exacerbaciones agudas y los días de enfermedad, definidos como días en cama, días de baja laboral o días en los que el paciente no pudo realizar sus actividades normales. También se determinaron los días en tratamiento con antibióticos. Las medidas de eficacia secundarias fueron la función pulmonar (volumen espiratorio forzado en un segundo, capacidad vital forzada y flujo espiratorio máximo) y los efectos adversos del tratamiento.

Se identificaron 27 ensayos clínicos que cumplían estos requisitos, 4 de los cuales fueron excluidos por no proporcionar información acerca de las medidas de eficacia primarias. Los otros 23 fueron incluidos en la revisión: 21 realizados en pacientes con bronquitis crónica y 2 en pacientes con neumopatía obstructiva crónica.

El uso habitual de mucolíticos se asoció a una reducción de 0,07 exacerbacionres por paciente y mes (intervalo de confianza del 95\%: 0,08 a 0,05; $P<$ $0,0001)$, lo cual representa un $29 \%$ menos que en el grupo de control. La razón de probabilidades (odds ratio) de no sufrir exacerbaciones durante el período de estudio fue de 2,22 (intervalo de confianza del 95\%: 1,93 a 2,54; $P<0,0001$ ) al comparar el tratamiento con mucolíticos frente al tratamiento con placebo. De acuerdo con estas cifras, sería necesario tratar a seis pacientes para que uno de ellos se mantuviera sin exacerbaciones durante el período de estudio. Al contrario de los demás mucolíticos estudiados, la isobutirilcisteína, un donante de grupos tiol, no proporcionó una reducción significativa de las exacerbaciones. El tratamiento mucolítico también redujo en 0,56 (intervalo de confianza del 95\%: $0,77$ a $0,35, P<0,0001)$ el número de días de enfermedad por paciente y mes, y en $0,53(0,76$ a 0,31 , $P<0,0001)$ el número mensual de días que los pacientes tomaron antibióticos. En los escasos estudios que aportaron datos sobre la función pulmonar, las diferencias entre los pacientes tratados con mucolíticos y con placebo fueron pequeñas y no significativas. Los acontecimientos adversos, generalmente leves y autolimitados, tuvieron una frecuencia similar en los pacientes tratados con placebo y en los tratados con mucolíticos (media de 0,77 acontecimientos por paciente en ambos grupos).

Estos resultados revelan que los mucolíticos reducen de forma moderada, pero significativa, las exacerbaciones y el número de días de enfermedad en pacientes con bronquitis crónica o neumopatía obstructiva crónica. Además, las exacerbaciones que se producen pueden no ser tan graves y los beneficios podrían ser mayores en los casos más graves. De cualquier forma, los beneficios son moderados y el tratamiento no parece tener una buena relación coste-eficacia. (Poole PJ, Black PN. Oral mucolytic drugs for exacerbations of chronic obstructive pulmonary disease: systematic review. BMJ 2001;322:1271-1274) 